\title{
Right atrial free-floating thrombus in a patient with acute pulmonary embolism
}

\author{
Kunal Mahajan, Prakash Negi, Sanjeev Asotra, Munish Dev
}

Department of Cardiology, Indira Gandhi Medical College, Shimla, Himachal Pradesh, India

\section{Correspondence to} Dr Kunal Mahajan, kunalmahajan442@gmail.com

Accepted 27 April 2016
CrossMark

To cite: Mahajan K, Negi P,
Asotra S, et al. BMJ Case
Rep Published online:
[please include Day Month
Year] doi:10.1136/bcr-2016-
215414

To cite: Mahajan $K$, Negi $P$, Rep Published online: (please include Day Month 215414

\section{DESCRIPTION}

A 66-year-old man was referred to us with a 3-day history of dyspnoea at rest. He had tachycardia (115/ min) with hypotension $(90 / 70 \mathrm{~mm} \mathrm{Hg})$ and elevated jugular venous pressure with clear lungs. Cardiac auscultation revealed no murmur. ECG revealed sinus tachycardia with a S1Q3T3 pattern. Transthoracic echocardiogram showed a highly echogenic ball-like mass in the dilated right atrium (RA) consistent with thrombus (figure 1). It was freely mobile and threatened to exit the RA into the right ventricle (RV) at any moment (video 1). McConnell's sign (RV dysfunction with characteristic sparing of the apex) was positive, suggesting pulmonary thromboembolism (PTE) (video 2). An emergent CT scan showed bilateral pulmonary emboli and a stalk free mass in the RA (figure 2A, B). Duplex scan of the lower limbs revealed no evidence of deep venous thrombosis (DVT). Alteplase was administered in a dose of $100 \mathrm{mg}$ as a continuous intravenous infusion over $2 \mathrm{~h}$. Thrombolysis led to complete lysis of the RA thrombus within an hour of administration (figure 3 and video 3). The patient became normotensive with improvement of the dyspnoea. There was no thrombolysis-related major or minor bleeding. Detailed work up for genetic and acquired causes of thrombophilia was negative. Right heart thrombi (RHT) have been reported in $4-18 \%$ of cases with acute PTE. ${ }^{1}$ In the largest study reported on

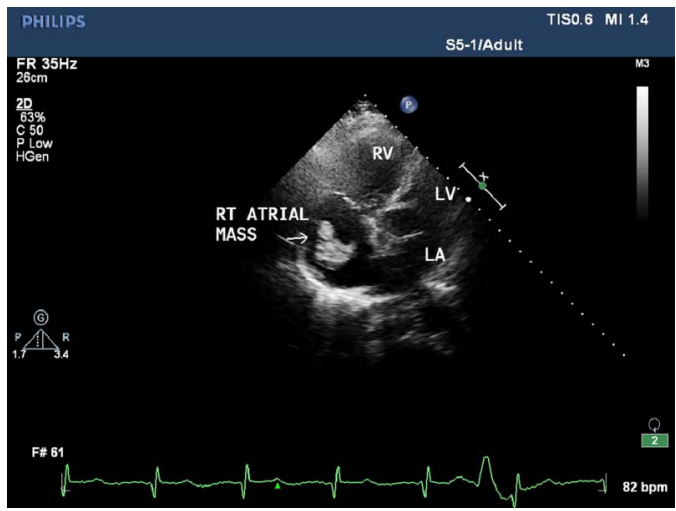

Figure 1 Transthoracic two-dimensional echocardiogram showing a ball-like thrombus in the right atrium. LA, left atrium; LV, left ventricle; RV, right ventricle.

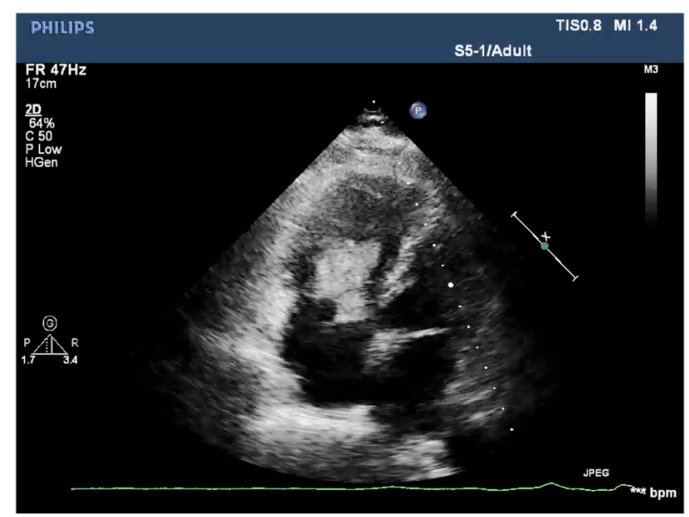

Video 1 Transthoracic echocardiogram, four-chamber-view, showing a freely mobile ball-like thrombus in the right atrium.

symptomatic free-floating RHT, 79\% of RHTwere in the RA, $16 \%$ were in the RV and 5\% were in both right heart chambers. ${ }^{2}$ They are round and ball-like when they form in the RA. Thrombolysis is convenient for patients with PTE and haemodynamic compromise or RV failure, and has the additional benefit of treating the PTE, RHT and associated DVT. Cardiac surgery is preferred for very large RHT, tricuspid occlusion, associated paradoxical embolism via patent foramen ovale transit, thrombolytic failure or contraindications to thrombolytic therapy. ${ }^{3}$

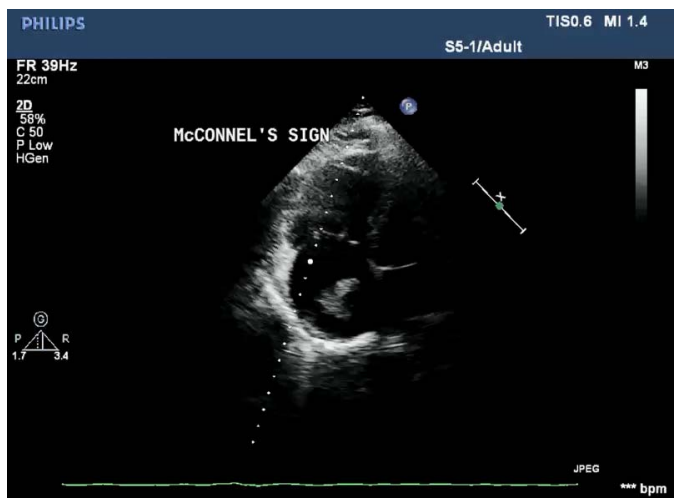

Video 2 Transthoracic echocardiogram demonstrating McConnell's sign. Note the hypokinesia of the right ventricular mid free wall with characteristic sparing of apex. 
Figure 2 (A) CT pulmonary angiogram showing filling defects, consistent with thrombi in the main pulmonary artery at the level of bifurcation, which are extending into bilateral pulmonary arteries (arrows). (B) Demonstration of a thrombus in the right atrium (arrow) on CT scan.
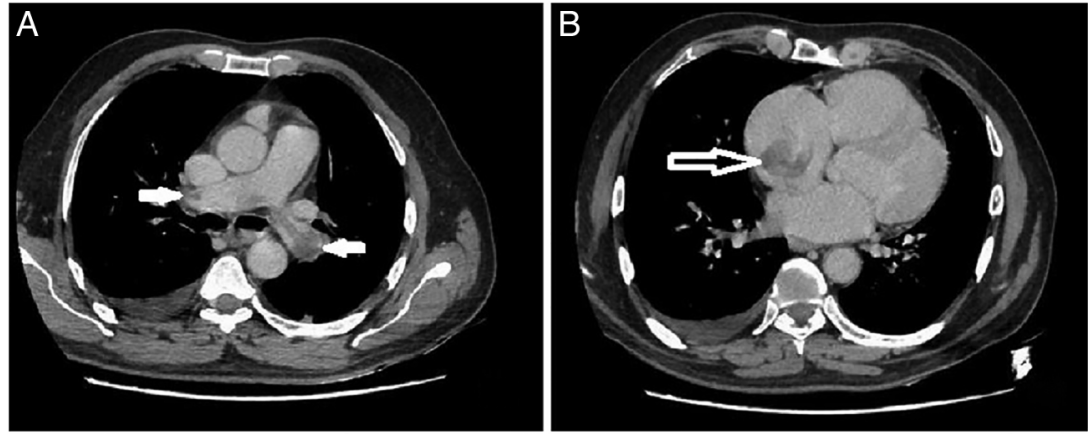

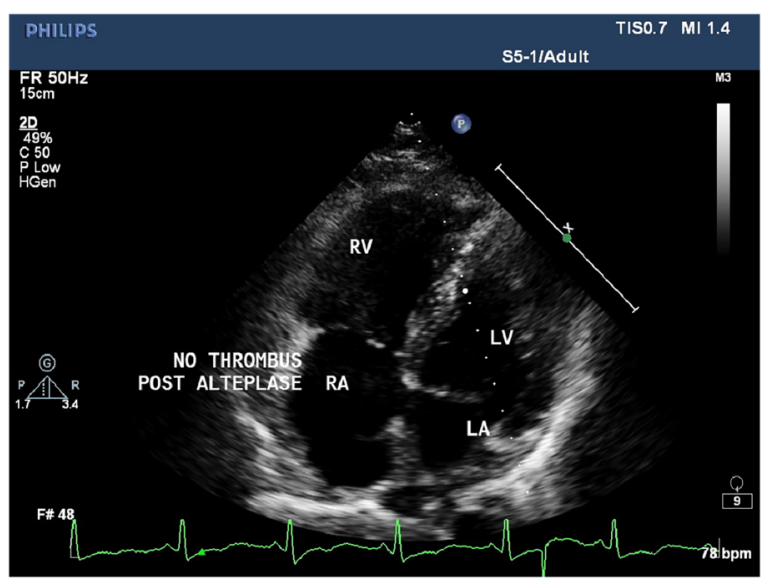

Figure 3 Two-dimensional echocardiogram performed immediately after thrombolysis, showing no residual thrombus in right atrium. $\mathrm{LA}_{t}$ left atrium; LV, left ventricle; $R A$, right atrium; $R V$, right ventricle.

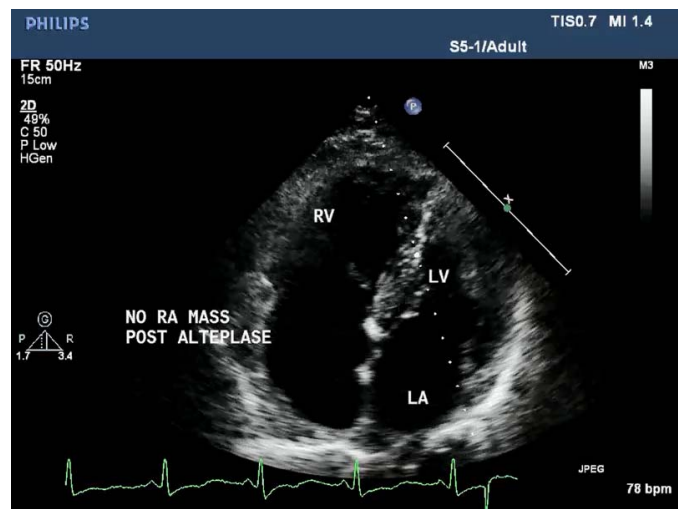

Video 3 Post-thrombolysis echocardiogram showing no residual thrombus.
Anticoagulation with heparin is reserved for haemodynamically stable patients who are not candidates for surgery/thrombolysis.

\section{Learning points}

- Right heart thrombi (RHT) have been reported in $4 \%$ to $18 \%$ of cases with acute pulmonary thromboembolism (PTE).

- They are seen more commonly in the right atrium (79\%) than the right ventricle $(16 \%)$.

- Thrombolysis is a better treatment modality, especially in haemodynamically unstable patients, as it treats RHT, PTE and associated deep venous thrombosis, simultaneously.

- In cases with very large RHT, or in patients with contraindication or failure with thrombolytics, surgery represents a convenient treatment option.

- Anticoagulation with heparin is reserved for haemodynamically stable patients who are not candidates for surgery/thrombolytic therapy.

Competing interests None declared.

Patient consent Obtained.

Provenance and peer review Not commissioned; externally peer reviewed.

\section{REFERENCES}

1 Torbicki A, Galié N, Covezzoli A, et al. Right heart thrombi in pulmonary embolism: results from the International Cooperative Pulmonary Embolism Registry. J Am Coll Cardiol 2003;41:2245-51.

2 Chartier L, Bera J, Delomez $\mathrm{M}$, et al. Free-floating thrombi in the right heart: diagnosis, management, and prognostic indexes in 38 consecutive patients. Circulation 1999:99:2779-83.

3 Jaff MR, McMurtry MS, Archer SL. Management of massive and submassive pulmonary embolism, iliofemoral deep vein thrombosis, and chronic thromboembolic pulmonary hypertension: a scientific statement from the American Heart Association. Circulation 2011;123:1788-830. 
Copyright 2016 BMJ Publishing Group. All rights reserved. For permission to reuse any of this content visit http://group.bmj.com/group/rights-licensing/permissions.

BMJ Case Report Fellows may re-use this article for personal use and teaching without any further permission.

Become a Fellow of BMJ Case Reports today and you can:

- Submit as many cases as you like

- Enjoy fast sympathetic peer review and rapid publication of accepted articles

- Access all the published articles

- Re-use any of the published material for personal use and teaching without further permission

For information on Institutional Fellowships contact consortiasales@bmjgroup.com

Visit casereports.bmj.com for more articles like this and to become a Fellow 